\title{
Evaluation of the immunological response of patients with childhood cancer treated with the personalized peptide vaccine for refractory soft tissue sarcoma: An early phase II study
}

\author{
Keiko Oda ${ }^{1}$, Yasuhiko Ito ${ }^{2}$, Akira Yamada ${ }^{3}$, Shigeru Yutani ${ }^{3}$, Kyogo Itoh $^{3}$, and Shuichi \\ Ozono $^{4}$ \\ ${ }^{1}$ College of Healthcare Management \\ ${ }^{2}$ Nagoya City University \\ ${ }^{3}$ Kurume University Cancer Vaccine Center \\ ${ }^{4}$ Kurume University
}

July 27, 2020

\begin{abstract}
We evaluated the peptide-specific immunoglobulin $\mathrm{G}(\mathrm{IgG})$ response and the safety of the personalized peptide vaccine in four children with refractory solid cancer. Although the pre-vaccination IgG responses were suppressed compared to those observed in children with upper respiratory tract infection, IgG levels against the vaccinated peptides after 12 times vaccination increased in all the three cases tested. Vaccination-related adverse effects were grade 1 injection-site local skin lesions. One patient had maintained remission for 37 months. The remaining three patients exhibited progressive disease. These results indicate the next steps in phase II studies considering their immune boosting effect and safety.
\end{abstract}

\section{Introduction}

Recent advances in pediatric multidisciplinary cancer therapy have resulted in long-term survival and full recovery in most patients. ${ }^{1,2}$ However, this impressive advancement is associated with late adverse effects owing to repeated intense treatments needed to achieve these results. One of the effects is the suppression of immune responses, which might in turn result in early recurrence ${ }^{3,4}$ or the development of secondary cancers. We developed a new approach for cancer immunotherapy, i.e., a personalized peptide vaccine (PPV), wherein peptides are selected from 31 different peptide candidates for individual patients on the basis of their secondary immune responses ${ }^{5-7}$. The PPV resulted in a longer overall survival (OS) in some patients with advanced adult cancers, as observed in phase II studies. ${ }^{8,9}$ Humoral responses against these 31 peptides - encoded by 15 different tumor-associated antigens - are detectable in healthy donors and cancer patients, and these levels were biomarkers for OS in not only vaccinated patients but also unvaccinated patients with advanced cancer. ${ }^{10,11}$ However, little is known about the immunological features of patients with refractory childhood cancer considering the tumor immunology, partly owing to the lack of an affordable assay system to measure the preexisting antitumor immunity. Accordingly, we conducted the current early phase II clinical study for patients with refractory childhood cancer to evaluate the possible application of the PPV as a new immunotherapy modality.

\section{Methods}

We conducted the current early phase II study of the PPV in children diagnosed with refractory solid cancer. The details of the protocol are given in Supplemental Appendix A1. The patient profiles are presented in Table 1. Four patients with pediatric sarcoma (alveolar rhabdomyosarcoma [ARMS], osteosarcoma, alveolar 
small cell tumor, and Wilms tumor, respectively) were enrolled in this study between October 2016 and March 2017. The safety profile was assessed by monitoring the occurrence of adverse events according to the National Cancer Institute Common Terminology Criteria for Adverse Events (version 4.0). For measuring the IgG responses to 31 peptides, IgG titers specific to the antigen peptides in the plasma were evaluated by using a bead-based multiplex Luminex assay, as previously described. ${ }^{5-12}$ We adopted "Fluorescence Intensity Unit (FIU)" for measuring peptide-specific IgG response associated with personalized vaccine. Komatsu reported the comparison between the IgG measured by ELISA and FIU ${ }^{12}$. The total sums of the immunoglobulins specific to each peptide were calculated. The protocol was approved by the Kurume University Ethical Committee and registered in the UMIN Clinical Trials registry (UMIN \# 000030249). The patient caregivers were given a full explanation of the protocol and provided informed consent before enrollment.

\section{Statistical analysis}

The 2-fold Wilcoxon test was used to examine the differences between young and adult patients with cancer and healthy controls. $P$-values $<.05$ were considered statistically significant.

\section{Results}

Although all the four patients showed grade 1 erythema or induration at the injection sites, none of them showed any other severe PPV-related adverse effects. The patients received the PPV a total of 22, 12, 12, and 7 times, respectively (Table 1). One patient (Case 1), who received the PPV alone, had a stage IV right sacrum-origin ARMS with lymph node metastasis (Figs. 1A and 1B). His tumor relapsed in the right kidney 12 months after the initial multidisciplinary therapy (Fig. 1C). After the completion of secondary chemotherapy, the patient visited our institute at the age of 5 years (2 months after the final chemotherapy) and was disease-free (Fig. 1D). He was administered the PPV alone 22 times for 22 months, and he has not shown any relapse 37 months since the PPV administration (Fig. 1E). Informed consent for using all these photographs was properly documented and obtained from the patient caregivers. The pre-vaccination immune response against the 31 peptide candidates in this patient was very low, but IgG levels against 2 of the 4 peptide vaccines were increased at the time of the $6^{\text {th }}$ vaccination, followed by a strong increase at the $18^{\text {th }}$ vaccination against all 4 peptides with a $>100$-fold increase vs. 2 peptides. Moreover, these higher IgG levels were maintained for nearly 12 months after the last vaccination (Fig. 1F). The remaining three cases (Cases 2, 3, and 4) had active tumors at the time of study enrollment, and had received chemotherapy combined with the PPV for 18, 9, and 6 months, respectively (Table 1). IgG boosting was observed in the post-vaccination plasma sample of all the three cases (cases 1 to 3 ) at the $12^{\text {th }}$ vaccination, but not at the $6^{\text {th }}$ vaccination from Case 4 who did not receive the $7^{\text {th }}$ vaccination owing to disease progression (Supplemental Table 2).

To better understand the immunological features of these four cases, their pre-vaccination IgG responses against the 31 peptides were compared to those of seven pediatric patients (age: 1-2 years) with upper respiratory tract infection (URI). IgG responses were detectable for only parts of the 31 peptides in the former cases, with total sums of 270,670, 203, and 596 FIU (Supplemental Table 2), whereas they were detectable for the vast majority of 31 peptides in the pediatric URI cases with total sums of 605, 1148, 860, 2053, 7378, 2268, and 5983, respectively (Supplemental Table 3). Subsequently, the total sum of the pre-vaccination IgG levels against 31 the peptides in the pre- or post-vaccination plasma samples of the four children was significantly lower $(\mathrm{p}=0.01)$ or higher $(\mathrm{p}>0.01)$ than that of the seven pediatric patients with URI, respectively (Fig. 1G).

\section{Discussion}

The results of the current early phase II study showed that pre-vaccination IgG levels against 31 peptide vaccine candidates in patients with relapsed childhood cancer were suppressed when compared with the URI group. This immunosuppression could be partly owing to adverse effects associated with repeated intensive treatments for relapsed childhood cancer. However, these suppressed immunological features were not only cancelled but rather strongly boosted by administering the PPV. This finding should be confirmed in a 
large-scale PPV study of childhood cancer with age-matched controls.

We treated only four patients using the PPV; hence, it is impossible to discuss or evaluate the clinical benefits of the PPV for childhood cancer. However, these findings might be useful for better designing the next step of the trial. Only one patient achieved long-term remission. The other three cases showed disease progression, regardless of the enhancement in the level of IgG antibodies to the vaccinated peptides in cases 2 and 3. Therefore, patients in remission, but not those with active metastatic tumors, might be more appropriate as candidates for PPV therapy.

Regarding case 1 that was successfully treated with the PPV, rhabdomyosarcoma is the most common soft tissue sarcoma in children, comprising $3.5 \%$ of cases among children aged $0-14$ years. ${ }^{13}$ A higher relapse rate (up to 50\%) was one of the features of ARMS. ${ }^{14-16}$ Furthermore, patients with ARMS with metastasis, similar to the patient reported herein, were reported to have dismal OS. ${ }^{17,18}$ The results of the current study suggest that the PPV has some consolidation effect on childhood solid tumors when the tumor is controlled at the time of entry, in agreement with the results of the effects of the WT1 peptide vaccination in five cases. ${ }^{19}$ Collectively, these results help in planning the next step of the phase II study of the PPV considering the immune boosting effect and safety.

Conflicts of interest : Itoh $\mathrm{K}$ received research funding from the Taiho Pharmaceutical Company. The remaining authors have nothing to declare.

Funding: The present study did not receive specific funding, but was performed as part of the Kurume University, Kurume Fukuoka, Japan, and in part by Sendai Kousei Hospital, Sendai, Japan

Acknowledgment : We thank Drs. Shigeki Shichijo, and Yasushi Ohtsu for their contribution in supporting the clinical study. We thank Junko Umezaki for supporting the patients and families as a responsible research nurse. We also appreciate all the patients with pediatric cancer and their families as well as all the children with infectious diseases of the respiratory tract.

\section{References.}

1. Pearson ADJ, Rossig C, Lesa G, et al . ACCELERATE and European medicines agency paediatric strategy forum for medicinal product development of checkpoint inhibitors for using combination therapy in paediatric patients. Eur J Cancer 2020; 127 : 52-66.

2. Siegel RL, Miller KD, Jemel A. Cancer statics. CA Cancer J Clin 2016; 66 : 7-30.

3. $P D Q{ }^{\circledR}$ Pediatric Treatment Editorial Board. PDQ cancer Information Summaries [Internet] . Late effect pf treatment. Bethesada (MD), National Cancer Institute 2002-2020.2020,Feb 4.

4. Leung W, Naele G, Behm F, et al. Deficient innate immunity, thymopoiesis, and gene expression response to radiation in survivors of childhood acute lymphoblastic leukemia. Cancer Epidemiology 2010; 34: 303-308

5. Takahashi R, Ishibashi Y, Hiraoka K, et al . Phase II study of personalized peptide vaccination for refractory bone and soft tissue sarcoma patients. Cancer Sci 2013; 104:1285-1294.

6. Iwasa S, Yamada Y, Heike Y, et al. PhaseIstudy of a new cancer vaccine of ten mixed peptides for advancer cancer patients. Cancer Sci 2016; 107: 590-600.

7. Takahashi R, Toh U, Iwakuma N, et al . Feasibility study of personalized peptide vaccination for metastatic recurrent triple-negative breast cancer patients. Breast Cancer Resarch 2014; 16: R70.

8. Noguchi M, Moriya F, Koga N et al . A randomized phase II clinical trial of personalized peptide vaccination with metronomic low-dose cyclophosphamide in patients with metastatic castration-resistant prostate cancer. Cancer Immunol Immunother 2016; 65 :151-60.

9. Suekane S, Ueda K, Nishihara K, et al . Personalized peptide vaccination as a second line treatment for metastatic upper tract urothelial carcinoma. Cancer Sci 2017; $108: 2430-2437$.

10. Sakamoto S, Yutani S, Schichijo S, et al . Immunological evaluation of personalized peptide vaccination for patients with histologically unfavorable carcinoma of unknown primary site. Cancer Immuno Immunother 2016; 65: 1223-1231. 
11. Sakamoto S, Matsueda S, Takamori S, et al . Immunological evaluation of personalized peptide vaccination for cancer patients with the HLA-A26 allele. Cancer Sci 2015; 106: 1257-1263.

12. Komatsu N, Shichijo S, Nakagawa M, et al . New multiplexed flow cytometric assay to measure antipeptide antibody: a novel tool for monitoring immune responses to peptides used for immunization. Scand J Clin Laborat Invest 2004; 64: 535-546.

13. Chen C, Garcia HK, Sheer M. Current and future treatment strategies for Rhabdomyosarcoma. Front Oncol 2019; 9:1958.

14. Camero S, Ceccarelli S, De Feris M, et al . PARP inhibitors affects survival, radiation susceptibility of human alveolar and embryonal rhabdomyosarcoma cell lines. J Cancer Res Clin Oncol 2019; 145:137152.

15. Stewart E, Mcvoy J, Wang H, et al . Identification of therapeutic targets-rhabdomyosarcoma integrated genomic, epigenomic, and proteomic analysis. Cancer cell 2018; 34 : 411-426.

16. Hawkins DS, Chi YY, Anderson R, et al. Additional vincristine and irinotecan to vincristine, dactinomycin, and cyclophosphamide does not improve outcome for intermediate-risk rhabdomyosarcoma : a report from the Children's Oncology Group. J Clin Oncol 2018; 36 : 2770-2777.

17. Kim JR, Yoon HM, Koh KM, et al. Rhabdomyosarcoma in children and adolescents : patterns and risk factors of distant metastasis. Am J Roentgenol 2017; 209 :409-416.

18. Amer KM, Thomson JE, Conquesta G, et al . Epidemiology, incidence and survival of rhabdomyosarcoma subtypes : SEER and ICES data base analysis. J Orthop Res 2019; 37: 2226-2230.

19. Hashii Y, Sato E, Ohta H, et al. WT1 Peptide immunotherapy for cancer in children and young adults. Pediatr Blood Cancer 2010; 55:352-355.

\section{Figure Legends}

Figure 1. A-E, Computed tomography (CT) scans and their date of examination.

A, Original tumor site. B, Metastasis to the peritoneal lymph node. C, Relapsed site (right kidney).D, CT findings before vaccination (no tumor). E, The latest CT findings (no tumor). Arrows indicate the location of the tumors. F, Immune response for vaccinated peptides. Arrows indicate the day of vaccination. "20XX" is the year of the first disease onset. G, Statistical analysis with the 2-fold Wilcoxon test.

Table 1: Abbreviation; VAC: vincristine, actinomycin D, cyclophosphamide chemotherapy, ICE: Ifosphamide, Carboplatin, Etoposide chemotherapy, IMRT: Intensity modulated radiation therapy, DXR: doxorubicin, MTX: methotrexate, IFO: ifosphamide, CDDP: cisplatinum, VDC: vincristine, doxorubicin, cyclophosphamide chemotherapy, IE: ifosphamide, etoposide chemotherapy, DD4A: d-actinomycin, doxorubicin, vincristine chemotherapy, PTK: Protein tyrosine kinases, TMZ: temozolomide, SD: stable disease, PD: progressive disease, OS: overall survival

Supplemental table 1: a: A3family, HLA-A3, A11, A31, and A33

Abbreviation: HLA, human leukocyte antgen ; TAA, tumor associated antigen

Supplemental table 2: Bold number indicated the selected peptide for vaccination

\section{Hosted file}

\selectlanguage\{english\}*200721Table_1_Profile\selectlanguage\{english\}*.docx available at https://authorea.com/users/346275/articles/472395-evaluation-of-the-immunologicalresponse-of-patients-with-childhood-cancer-treated-with-the-personalized-peptidevaccine-for-refractory-soft-tissue-sarcoma-an-early-phase-ii-study 

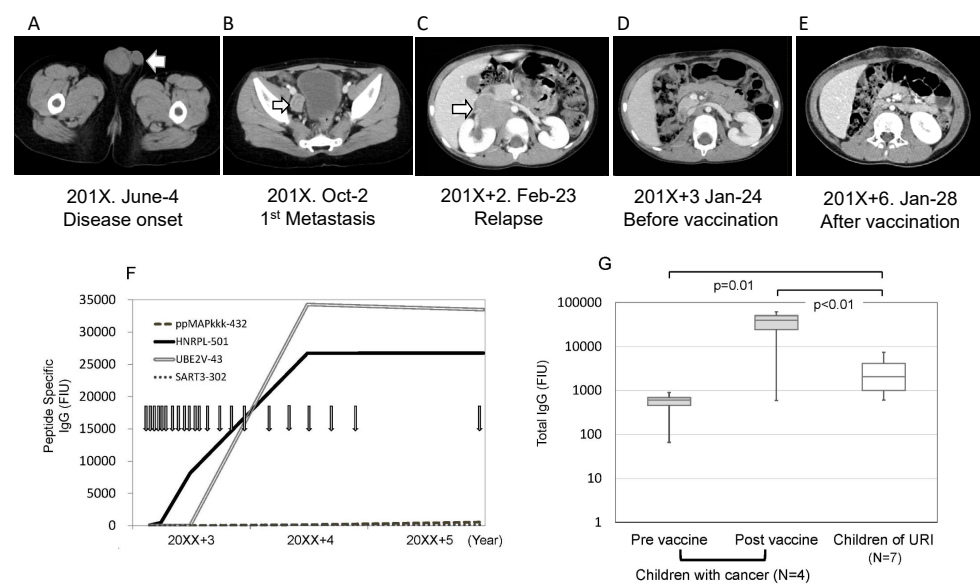

Figure 1 